\title{
Selectin-Mediated Interactions Regulate Cytokine Networks and Macrophage Heme 0xygenase-1 Induction in Cardiac Allograft Recipients
}

\author{
Ana J. Coito, Gray D. Shaw, Jiye Li, Bibo Ke, Jeffrey Ma, Ronald W. Busuttil, and \\ Jerzy W. Kupiec-Weglinski \\ Dumont-UCLA Transplant Center (AJC, JL, BK, JM, RWB, JWK-W), Department of Surgery, UCLA School of \\ Medicine, Los Angeles, California; and Genetics Institute/Wyeth Research (GDS), Cambridge, Massachusetts
}

\begin{abstract}
SUMMARY: Host sensitization to major histocompatibility complex (MHC) antigens is among the most critical of problems facing heart transplantation. Selectins are postulated to mediate the early adhesive events in the recruitment of leukocytes at the allograft site. We investigated the significance of selectin-P-selectin glycoprotein ligand-1 (PSGL-1)-mediated in vivo interactions in the immune cascade leading to rejection of cardiac allografts in skin presensitized rats. Infusion of a soluble recombinant form of PSGL-1 (rPSGL-lg) during skin graft-mediated sensitization prevented Day $1.0 \pm 0.1$ "accelerated" rejection in sensitized rat recipients, and prolonged cardiac allograft survival to Day $3.8 \pm 1.0(p<0.001)$. This therapy significantly depressed serum IgM levels and decreased intragraft expression of Th1 type cytokines (IL-2 and IFN- $\gamma$ ) as well as of IL-1 $\beta$ and MCP-1, as compared with controls, without affecting the initial number of infiltrating mononuclear cells (MNC). A profound decrease in graft-infiltrating MNC was recorded at 24 hours in rPSGL-Ig-treated rats. The expression of heme oxygenase-1 (HO-1), an inducible heat shock protein 32 that protects against oxidative cell/tissue injury, was found in approximately 14-fold higher levels in the rPSGL-Igtreated recipients as compared with controls. The HO-1 overexpression in rPSGL-lg-treated hosts, primarily by infiltrating macrophages, was accompanied by virtual absence of myocardial infarcts and decreased frequency of TUNEL + cells at the graft site. Moreover, down-regulation of HO-1 expression by zinc protoporphyrin, an $\mathrm{HO}-1$ antagonist, decreased expression of antiapoptotic Bag-1 molecule in recipients conditioned with rPSGL-lg. Thus, the blockade of selectin-PSGL-1 interactions depresses intracardiac allograft expression of Th1 type cytokines, and might inhibit the differentiation of Th1 type cells. In addition, it up-regulates $\mathrm{HO}-1$ expression and protects against myocardial infarction and apoptosis. Hence, this study reports on a previously unrecognized role of selectin-PSGL-1-mediated interactions after in vivo alloantigenic challenge. (Lab Invest 2002, 82:61-70).
\end{abstract}

\begin{abstract}
A multistep series of adhesive and signaling events regulates the recruitment of leukocytes to major histocompatibility complex (MHC)-incompatible organ allograft (Coito et al, 1994). The selectin family of adhesion receptors, L-, E-, and P-selectin, and their carbohydrate ligands, are considered the primary mediators of leukocyte tethering and rolling on the surface of endothelial cells (McEver and Cummings, 1997). Indeed, studies in selectin-deficient mice have not only confirmed the role of these membraneanchored C-type lectins in leukocyte rolling, but also provided evidence indicating an overlapping function of the selectins in the regulation of inflammatory processes (Frenette et al, 1996; Homeister et al, 1998). L-selectin is expressed on most leukocytes. E- and P-selectins are expressed on activated endothelial cells, and P-selectin is also expressed on activated platelets. Selectins bind
\end{abstract}

\section{Received September 27, 2001}

This work was supported by American Heart Association Grant 0030006N (AJC), NIH Grants RO1 AI23847, RO1 AI42223 (JWK-W), and The Dumont Research Foundation.

Address reprint requests to: Dr. Ana J. Coito, The Dumont-UCLA Transplant Center, Rm. 77-120 CHS, Box 957054, Los Angeles, CA 90095.

E-mail:acoito@mednet.ucla.edu with relatively high affinity to only a few glycoproteins on leukocytes or endothelial cells (McEver and Cummings, 1997). P-selectin glycoprotein ligand-1 (PSGL-1), a disulfide-bonded homodimeric mucin-like glycoprotein expressed virtually on all leukocytes, is the best-defined ligand of the selectin family. Although PSGL-1 was originally discovered as the principal ligand for $\mathrm{P}$-selectin, it was subsequently demonstrated that this glycoprotein ligand binds to all three P-, E-, and L-selectin members (McEver and Cummings, 1997).

We have shown that the blockade of PSGL-1-mediated interactions with a soluble recombinant form of PSGL-1 (sPSGL-1) inhibits recruitment of polymorphonuclear leukocytes (PMN) and mononuclear cells (MNC) in rat models of kidney (Takada et al, 1997) and liver ischemia/reperfusion (I/R) injury (Dulkanchainun et al, 1998), resulting in significantly less organ dysfunction. There is evidence that functional selectin ligands are predominantly expressed on Th1 rather than Th2 cells (Lim et al, 1999). In organ transplantation, host immune responses seem to be qualitatively distinct depending on whether there is predominance of Th1 or Th2 cells at the graft site (Zhai and Kupiec-Weglinski, 1999).

The present study is the first to elucidate the significance of selectin-PSGL-1-mediated in vivo interactions 
in sensitized cardiac allograft recipients. The blockade of selectin-mediated interactions, with a soluble recombinant form of the PSGL-1 (rPSGL-Ig), favored inhibition of Th1 differentiation and decreased expression of the monocyte chemoattractant protein (MCP-1) in a model of accelerated cardiac allograft rejection in presensitized rats. Moreover, rPSGL-Ig therapy induced intragraft expression of heme oxygenase-1 (HO-1), a heat shock protein (hsp32) that protects against oxidative tissue injury, prevented the development of myocardial infarcts, and protected against myocardial apoptosis.

\section{Results}

\section{Treatment with rPSGL Ig Prolongs Cardiac Allograft Survival in Sensitized Rats}

$(\mathrm{LEW} \times \mathrm{BN}) \mathrm{F} 1$ cardiac allografts are rejected in less than 24 hours in Lewis (LEW) rats sensitized with Brown Norway (BN) skin grafts 7 days earlier (Day -7 ) (Kupiec-Weglinski et al, 1991). Treatment with rPSGL-Ig during the effector phase (postcardiac engraftment) failed to affect the 24-hour rejection response (Table 1). In contrast, cardiac allograft survival in rats that received a course of rPSGL-Ig during the sensitization phase (between skin and cardiac engraftment) was significantly increased to Day $3.8 \pm 1.0$, as compared with controls (Day $1.0 \pm 0.1, p<0.001$; Table 1). Extended pre- and post-transplant rPSGL-lg treatment did not improve further allograft survival. Animals conditioned with rPSGL-lg during the sensitization phase (Day -7 to -1 ) were used in these studies.

\section{Treatment with rPSGL-Ig Depresses Serum IgM Allo-Ab Levels}

To define the donor-specific allo-Ab responses elicited by $(\mathrm{LEW} \times \mathrm{BN}) \mathrm{F} 1$ cardiac allografts in $\mathrm{LEW}$ rats sensitized with BN skin grafts, serum was harvested from recipients preconditioned with PPSGL-Ig or PBS at Day $-5,-3,0$ (the day of cardiac engraftment), and at Day $+1,+4,+7,+14$, and +21 thereafter. Serum samples were then tested for IgM and IgG binding to BN lymph node target cells by flow cytometry. In control recipients, IgM allo-Ab levels increased considerably by Day 0 and remained elevated until Day 7 post-transplant (Fig. 1A). In contrast, in recipients treated with rPSGL-Ig, the anti-donor IgM response peaked at Day 4, that is, around the time of ultimate

Table 1. The Effects of rPSGL-Ig Therapy on the Survival of LBNF1 Cardiac Allografts in Skin Presensitized LEW Rats

\begin{tabular}{lccc}
\hline \multicolumn{1}{c}{ Group } & $\begin{array}{c}\text { Cardiac } \\
\text { allograft } \\
\text { survival (days) } \\
\text { (mean } \pm \text { SD) }\end{array}$ & $n$ & $\begin{array}{c}\text { Significance } \\
(p<)\end{array}$ \\
\hline Control & $1.0 \pm 0.1$ & 6 & \\
rPSGL-Ig (Day 0 to +1$)$ & $1.0 \pm 0.3$ & 5 & 0.37 \\
rPSGL-Ig (Day -7 to -1$)$ & $3.8 \pm 1.0$ & 6 & 0.001 \\
rPSGL-Ig (Day -7 to +2$)$ & $3.3 \pm 0.5$ & 3 & 0.007 \\
\hline
\end{tabular}

allograft rejection (Fig. 1A). At both Day 0 and +1 the IgM allo-Ab levels were significantly decreased in the rPSGL-Ig-treated group as compared with controls ( $p$ $<0.03$ and $p<0.05$, respectively). No significant differences in anti-donor IgG allo-Ab levels between treated and control groups were detected (Fig. 1B).

\section{rPSGL-Ig Therapy Arrests Intramyocardial Leukocyte Infiltration Without Affecting P-Selectin Expression}

rPSGL-Ig treatment during the sensitization phase extended cardiac allograft survival from less than 24 hours to more than 3 days. We analyzed intragraft cellular infiltration at 6 hours and 24 hours post-transplant $(n=$ $3-5 /$ group). At 6 hours, cardiac allografts from rPSGLIg-treated and control recipients showed relatively normal histology with only slight interstitial edema and sparse intramyocardial MNC infiltration (Table 2). By 24 hours, control grafts exhibited severe interstitial edema, large areas of myocardial infarction, and dense MNC infiltration. The infiltrating cells consisted predominantly of T lymphocytes, CD4+ cells, CD8+ cells, macrophages, and IL-2R+ cells (Table 2; Fig. 2, A to C). In contrast, the 24-hour grafts from rPSGL-Ig-treated recipients revealed good preservation of myocardial architecture, with significantly less intramyocardial T cell $(p<$ $0.001)$, CD4 + cell $(p<0.04)$, CD8 + cell $(p<0.0001)$, macrophage $(p<0.001)$, and IL-2R+ cell $(p<0.01)$ infiltration, as compared with respective controls (Table 2; Fig. 2, $\mathrm{F}$ to $\mathrm{H}$ ). As $\mathrm{P}$-selectin represents the principal ligand for PSGL-1, we then investigated whether administration of rPSGL-lg depressed P-selectin expression. The expression of $\mathrm{P}$-selectin by vascular graft endothelium was comparable at 6 hours $( \pm /+)$ and 24 hours $(++/+++)$ after transplantation in both treated and untreated groups, suggesting that rPSGL-lg blocks the interaction rather than regulates $\mathrm{P}$-selectin molecule expression. Moreover, rPSGL-lg therapy failed to affect the expression of other vascular adhesion molecules, with both control and treated 24-hour grafts showing moderate to high-level expression of VCAM-1 $(+/++)$ and ICAM-1 $(++/+++)$.

\section{rPSGL-Ig Therapy Modifies Intragraft Cytokine Networks}

We have employed competitive RT-PCR and immunohistology to analyze cytokine gene and protein expression patterns in cardiac allografts. Interestingly, despite comparable numbers of MNC infiltrating cardiac allografts at 6 hours in both rPSGL-Ig and control recipients, the cytokine networks were highly affected. At 6 hours, the mRNA levels coding for IL-1 $\beta(p<$ $0.04)$, IL-2 ( $p<0.02)$, IFN- $\gamma(p<0.005)$, and MCP-1 ( $p$ $<0.05)$ were significantly depressed in treated recipients as compared with the respective controls (Fig. 3). At 24 hours, intragraft expression of IL-2 mRNA remained depressed in rPSGL-Ig-treated hosts, as compared with controls ( $p<0.004$; Fig. 3). The immunohistochemical analysis confirmed the translation of mRNA into the relevant proteins (Fig. 2).

Figure 3 also shows that at 6 hours, the CD3 and CD25 mRNA levels were comparable in both recipient 


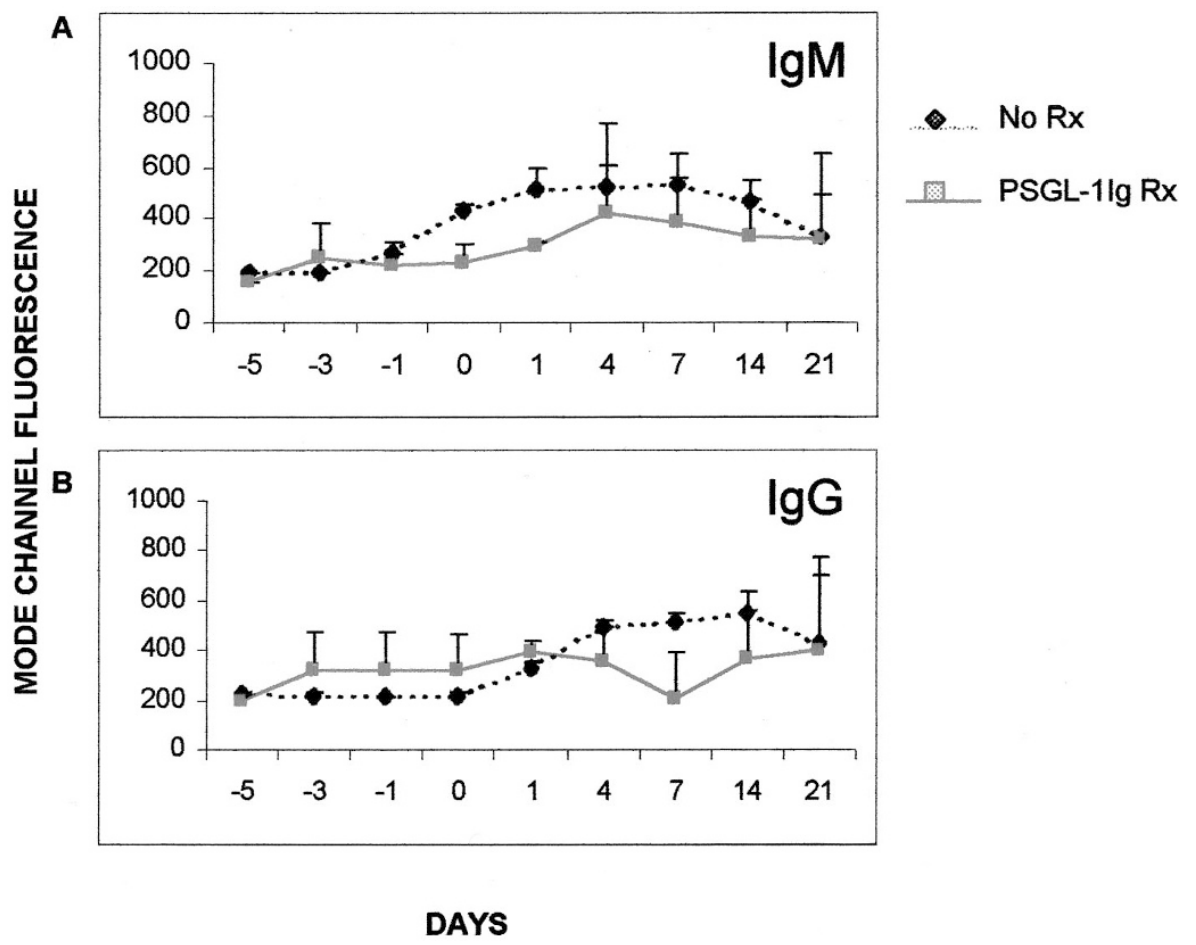

\section{Figure 1.}

Flow cytometry analysis of circulating IgM and IgG allo-Ab levels in untreated (no $R x$ ) and rPSGL-Ig-treated (rPSGL-Ig Rx) presensitized cardiac allograft recipients. In control recipients, IgM allo-Ab levels increased at Day 0 to 1 and remained elevated until Day 7 post-transplant, contrasting with rPSGL-Ig-treated recipients in which IgM responses peaked at Day 4, ie, around the time of ultimate allograft rejection (A). No significant difference was found in the IgG allo-Ab response between control and treated recipients (B).

Table 2. Immunohistochemical Analysis of Intramyocardial Infiltrating MNC in Cardiac Allografts

\begin{tabular}{|c|c|c|c|c|c|}
\hline \multirow[b]{2}{*}{ Feature } & \multicolumn{2}{|c|}{ No $R x$. } & \multicolumn{2}{|c|}{ rPSGL-Ig Rx. } & \multirow{2}{*}{$\frac{\text { rPSGL-Ig }+ \text { ZnPP Rx. }}{24 \text { hours }}$} \\
\hline & 6 hours & 24 hours & 6 hours & 24 hours & \\
\hline T cells & $5.0 \pm 2.3$ & $67.0 \pm 3.0$ & $3.3 \pm 2.1$ & $18.2 \pm 10.9$ & $35.0 \pm 4.2$ \\
\hline CD4 + cells & $33.0 \pm 2.0$ & $40.5 \pm 11.0$ & $24.5 \pm 7.5$ & $20.6 \pm 5.5$ & $26.0 \pm 1.4$ \\
\hline CD8 + cells & $1-2$ & $>200$ & $1-2$ & $34.2 \pm 18.5$ & $85.0 \pm 16.9$ \\
\hline Mono/Mac & $24.8 \pm 3.2$ & $>200$ & $22.0 \pm 0.6$ & $56.5 \pm 32.0$ & $96.5 \pm 2.1$ \\
\hline IL-2R + cells & $1.0 \pm 1.0$ & $42.5 \pm 4.5$ & $0.6 \pm 0.6$ & $13.7 \pm 6.6$ & $32.5 \pm 7.0$ \\
\hline
\end{tabular}

groups. At 24 hours, in agreement with our intragraft MNC infiltration analysis (Table 2; Fig. 2), mRNA levels coding for CD3 $(p<0.031)$ and CD25 $(p<0.05)$ were profoundly depressed in the rPSGL-Ig-treated recipients, compared with controls (Fig. 3).

\section{HO-1 is Induced by Intragraft Macrophages in rPSGL-Ig-Conditioned Recipients}

The expression of $\mathrm{HO}-1$ at 6 hours post-transplant was negligible in both rPSGL-lg and control groups. By 24 hours, however, cardiac allografts from treated recipients showed up-regulation of $\mathrm{HO}-1$ expression as compared with controls (Fig. 4A). Indeed, Western blotassisted densitometry measurements indicated 14-fold higher levels of HO-1 in rPSGL-lg-treated recipients $(p<$ 0.001 ). To identify the source of $\mathrm{HO}-1$ in cardiac allografts, we performed immunocytochemistry studies. In cardiac grafts of both treated and untreated groups, only a few vessels were sparsely positive for $\mathrm{HO}-1$ at 24 hours after transplantation. We identify the infiltrating MNC cells as the primary source of $\mathrm{HO}-1$ in the grafts. In rPSGL-Ig-treated grafts the infiltrating MNC were strongly $\mathrm{HO}-1$ positive, whereas in control grafts $\mathrm{MNC}$ were barely $\mathrm{HO}-1$ positive (Fig. 4, $\mathrm{B}$ and $\mathrm{C}$ ). The $\mathrm{HO}-1-$ positive cells usually had the convoluted shape typical of macrophages. We also performed a comparative study in sequential section stained for ED1 + and HO-1 + cells, and we could identify macrophages as a predominant source of HO-1.

\section{rPSGL-Ig Therapy Protects Against Apoptosis in Infarcted Myocardial Areas}

We then performed the terminal deoxynucleotidyl transferase (TdT) nick-end labeling (TUNEL) assay to assess whether rPSGL-Ig therapy protected against apoptosis. 

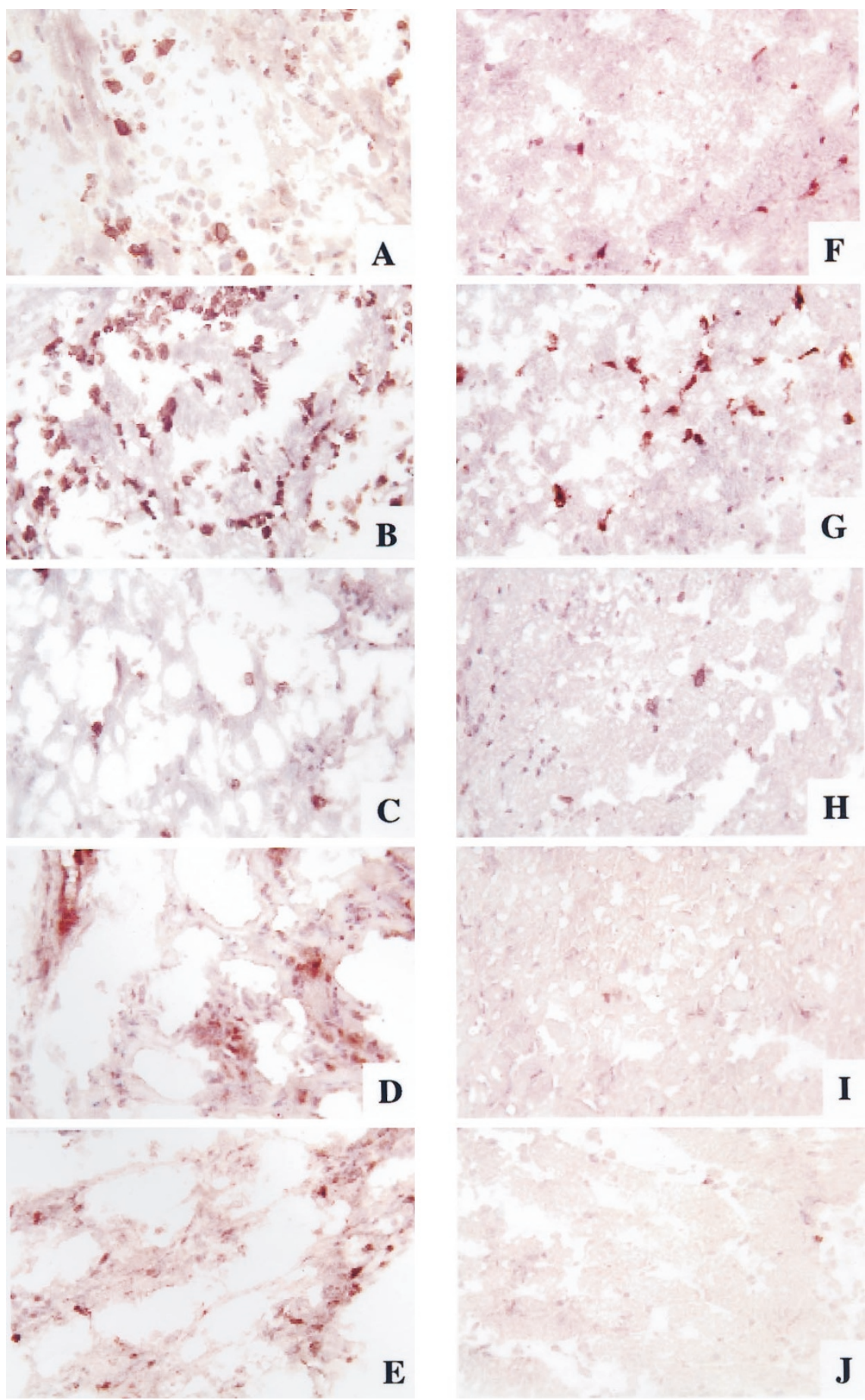

C

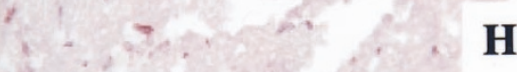

$\mathbf{H}$
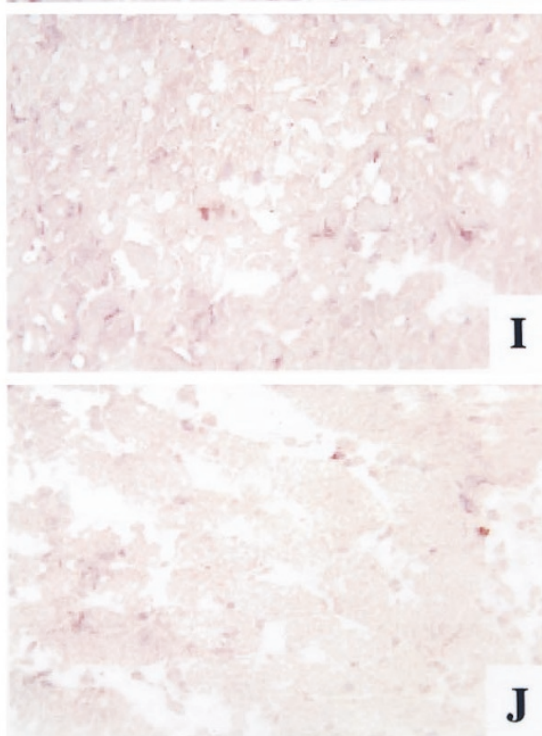

Figure 2.

Immunohistochemical analysis of infiltrating cells/cytokines in rat cardiac allografts (24 hours). Representative analysis of cardiac allografts in skin sensitized, otherwise untreated, recipients ( $A$ to $E$ ), and after rPSGL-Ig treatment ( $F$ to J). Rejecting grafts were characterized by an elevated number of infiltrating $T$ cells ( $A$ ) and macrophages (B), contrasting with their decreased number in allografts from rPSGL-Ig-treated hosts ( $\mathrm{F}$ and $\mathrm{G}$, respectively). In addition, rPSGL-Ig therapy virtually abolished intragraft cell activation markers, such as IL-2R+ cells (H), IL-2 (I), and IFN- $\gamma(\mathrm{J})$. Moderate to high numbers of IL-2 R+ cells (C), IL-2 (D), and IFN- $\gamma(E)$ were found in control cardiac allografts. Original magnification, $\times 200$.

No TUNEL-positive cells were observed in cardiac grafts of rPSGL-Ig-treated or control recipients at 6 hours post-transplant. However, unlike in the rPSGL-lgtreated grafts that remained negative for TUNEL staining (Fig. 4E), increased numbers of TUNEL-positive myocytes were readily detectable by 24 hours in infarcted areas and in some vessels of control grafts (Fig. 4D).
Zinc Protoporphyrin (ZnPP) Increases T Cell Infiltration/Activation and Down-Regulates Bag-1 Expression in rPSGL-Ig-Conditioned Hosts

To determine whether the beneficial effect of rPSGL-lg therapy was $\mathrm{HO}-1-$ mediated, we administrated $\mathrm{ZnPP}$, a competitive inhibitor of $\mathrm{HO}-1$ activity, to rPSGL-lg- 
CD3
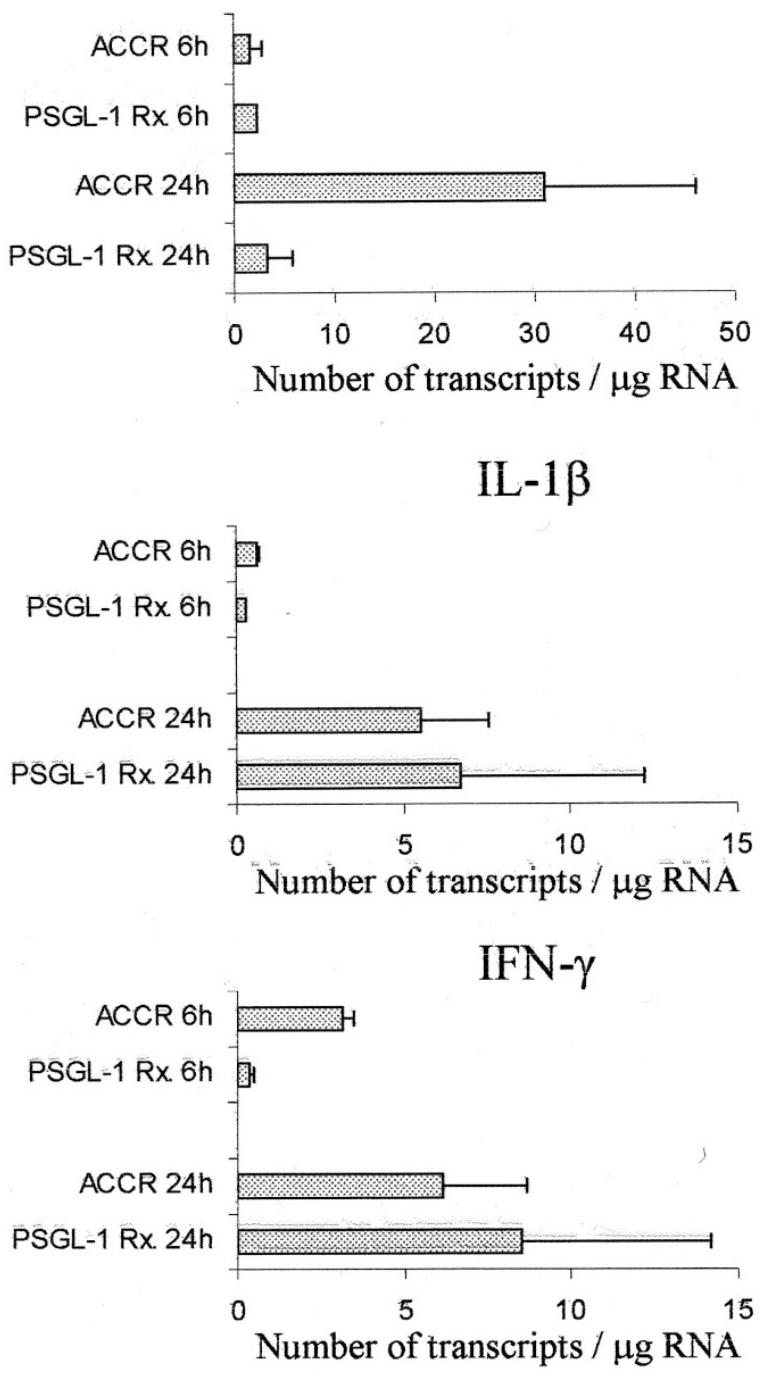

MCP-1

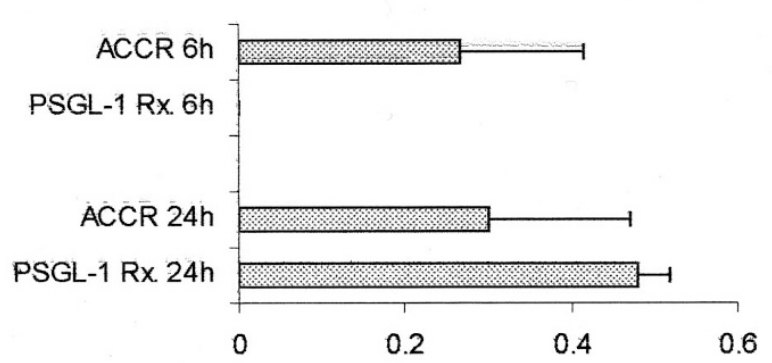

Ratio MCP-1 / $\beta$ Actin mRNA
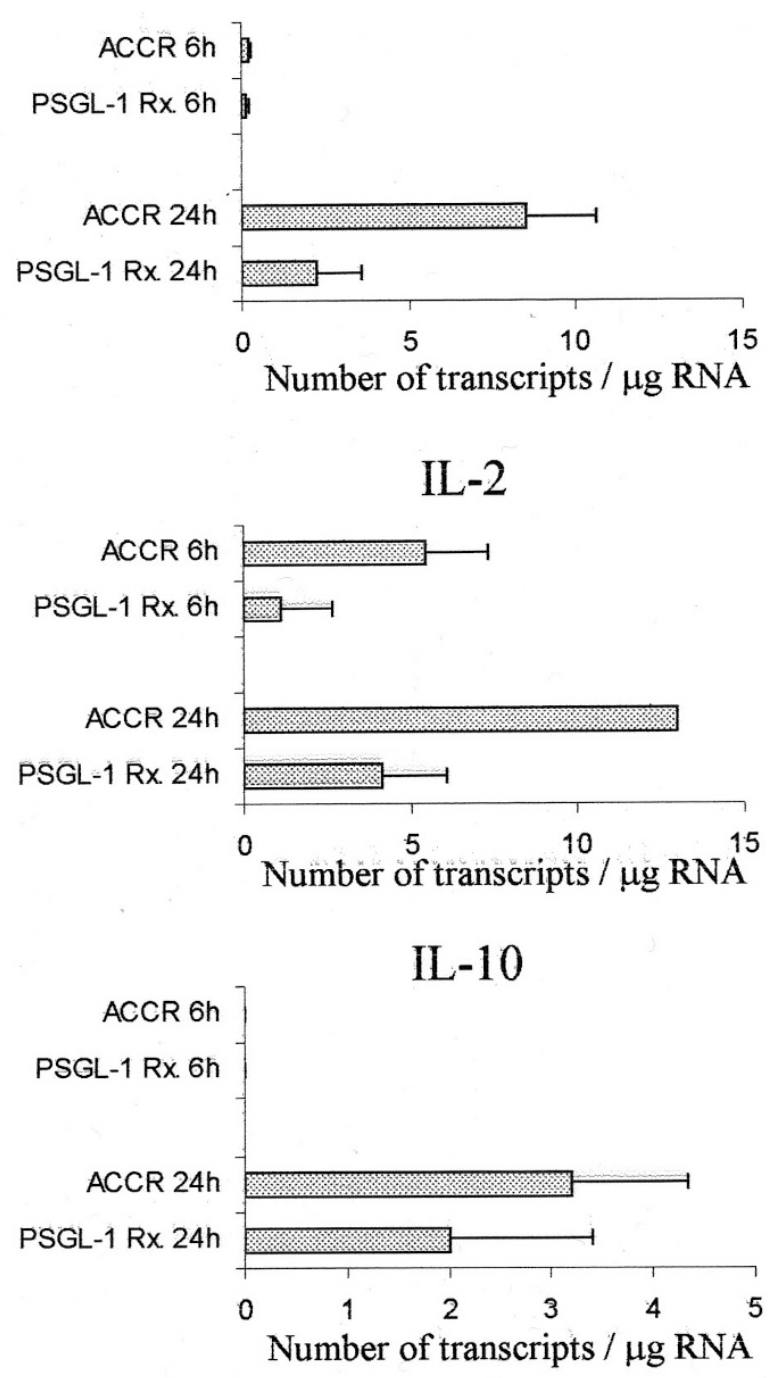

RANTES

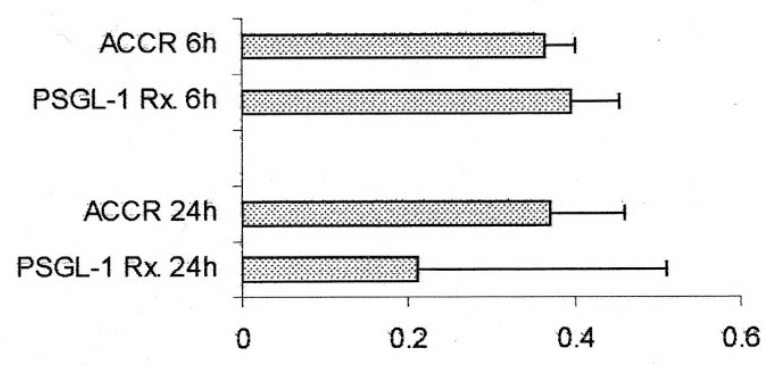

Ratio RANTES / $\beta$ Actin mRNA

Figure 3.

Competitive template RT-PCR-assisted analysis of intragraft cytokines in untreated ( $n o R x)$ and rPSGL-lg-treated ( $r P S G L-\lg R x)$ cardiac allograft recipients. The levels of CD3 and CD25 mRNA were comparable in both recipient groups at 6 hours post-transplant. In contrast, rPSGL-Ig therapy significantly decreased the expression of mRNA coding for IL-1 $\beta(p<0.04)$, IL-2 $(p<0.02)$, IFN- $\gamma(p<0.005)$, and MCP-1 $(p<0.05)$. IL-10 was almost absent in all cardiac grafts at this initial time point. At 24 hours, control allografts were characterized by elevated CD3, CD25, and IL-2 mRNA levels, as compared with those in the rPSGL-Ig-treated group $(p$ $<0.03, p<0.05, p<0.004$, respectively). 

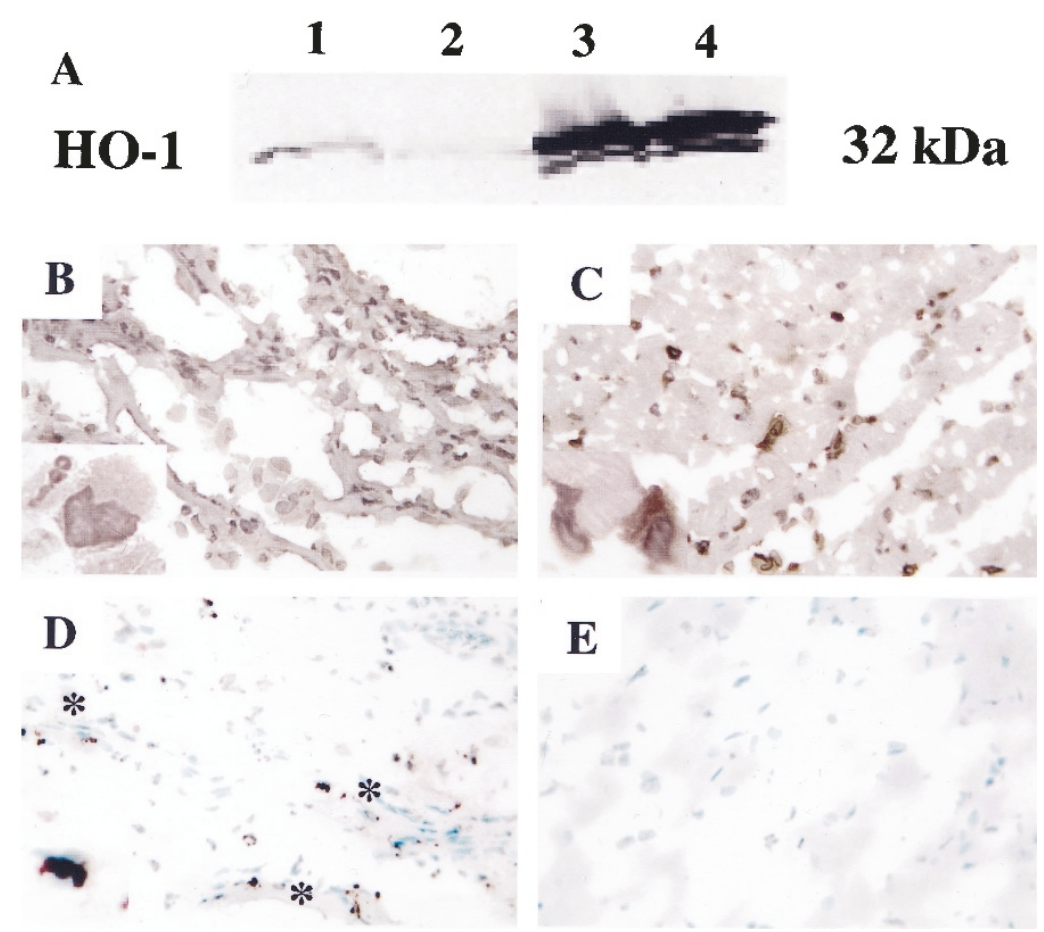

Figure 4.

Western blot-assisted detection of $\mathrm{HO}-1$ and of TUNEL-assisted staining of apoptotic cells in rat cardiac allografts (24 hours). A, rPSGL-Ig therapy up-regulated detection of H0-1 expression (Lanes 3 and 4), as compared with control grafts (Lanes 1 and 2). B, Despite dense infiltration of control grafts by MNC, including macrophages, cells are faintly positive for $\mathrm{HO}-1$ staining. C, An intense staining for $\mathrm{HO}-1$ by infiltrating macrophages in cardiac allografts from rPSGL-Ig-treated rats. Enlargements of cellular staining are provided in the lower left inserts. D, TUNEL + staining in both myocardium and vessels of control grafts. Areas of positive labeling are identified by asterisk $\left(^{*}\right)$, and enlargement of cellular staining is provided in lower left insert. E, In contrast, no TUNEL+ cells were detected in the grafts of rPSGL-Ig-treated recipients. Original magnification, $\times 200$.

treated recipients. We have shown that ZnPP exerts its biological effects quickly by down-regulating $\mathrm{HO}-1$ protein/activity in cardiac grafts (Katori et al, in press). Adjunctive administration of ZnPP to rPSGL-lg-treated recipients resulted in a loss of myocardial preservation and a significant increase of T cell $(p<0.03)$, CD8 + cell ( $p<0.04)$, and IL-2R+ cell $(p<0.05)$ infiltration as compared with recipients treated with rPSGL-lg alone (Table 2). No significant effect was observed in CD4+ cell and macrophage infiltration after ZnPP administration (Table 2). In addition, to decreased T cell infiltration/ activation, adjunctive ZnPP therapy markedly decreased the expression of antiapoptotic Bag-1 protein (mean densitometric ratio rPSGL-lg + ZnPP : rPSGL-lg = 0.6; $n$ $=3$ ). Moreover, intragraft expression of proapoptotic caspase-3 (p20), undetectable otherwise in recipients treated with PSGL-Ig alone, was moderately increased by densitometry (net intensity: $1567 \pm 343$ ) after adjunctive $\mathrm{ZnPP}$ therapy.

\section{Discussion}

We report here the results of our studies on the role of selectin-PSGL-1-mediated interactions in a welldefined model of cardiac transplantation in sensitized rat recipients. Treatment with rPSGL-Ig before cardiac engraftment (a) significantly prolonged cardiac allograft survival; (b) profoundly depressed intragraft expression of type- 1 cytokines (IL-2 and IFN- $\gamma$ ), as well as of IL-1 $\beta$, and MCP-1; (c) significantly depressed the number of infiltrating MNC; (d) induced intragraft overexpression of $\mathrm{HO}-1$; and (e) protected against the development of myocardial infarcts and apoptosis.

Administration of rPSGL-Ig during the sensitization phase ameliorated 24-hour rejection and significantly prolonged the survival of cardiac allografts to over 3 days. Very few therapeutic modalities are effective in this stringent sensitized rat cardiac transplant model, which makes rPSGL-lg-induced prolongation of allograft survival of particular significance.

A growing number of reports have been showing the crucial role for selectin-mediated interactions on leukocyte tethering and rolling on endothelial cells (Frenette et al, 1996). We observed that administration of rPSGL-Ig to cardiac recipients significantly decreased the expression of type- 1 cytokines, compared with controls, consistent with their role in the rejection cascade (Zhai and Kupiec-Weglinski, 1999). Interestingly, at 6 hours post-transplantation, despite differences in cytokine expression in cardiac grafts of treated versus control recipients, grafts from both groups showed comparable $\mathrm{T}$ cell infiltrate and CD3 mRNA levels. These results might favor the notion that rPSGL-Ig preferentially inhibited the recruitment of Th1-like cells to the graft site. Indeed, the selectin ligands are preferentially expressed on Th1 cells and play a key role in their recruitment to sites of inflammation (Austrup et al, 1997; Lim et al, 1999). Supporting this concept is our observation of up-regulated 
P-selectin expression on the vascular endothelium in the first few hours post-transplantation. If the inhibition of lymphocyte rolling by rPSGL-Ig can explain, at least in part, our results, it does not answer the question of why treatment of sensitized recipients with rPSGL-Ig failed when given immediately after heart transplantation. It seems logical to raise the assumption that selectin-mediated interactions may play a role in the $\mathrm{T}$ cell costimulatory pathway favoring the generation of effector Th1 cells. The immunosuppressive properties of rPSGL-Ig might, to a certain extend, contribute to the inhibition of Th1 cell differentiation during the sensitization phase. The expression of $\mathrm{P}$ and E-selectin ligands is controlled by the activity of $\alpha(1,3)$-fucosyltransferases that modify carbohydrates on PSGL-1 and other surface receptors (Fuhlbrigge et al, 1997). Moreover, the expression of one enzyme of the $\alpha(1,3)$-fucosyltransferase family, FucTVII, is highly linked to Th1 cell differentiation (Wagers et al, 1998). The hypothesis that a primary role of the rPSGL-Igmediated therapy is to inhibit Th1 differentiation provides a better justification of why postcardiac transplant therapeutic approach failed to affect the under 24-hour rejection in our model. At that time, the effector $T$ cells triggered by skin graft-driven host immune response are already differentiated and ready to reject cardiac transplants in an accelerated manner. In support of our theory that the rPSGL-Ig-mediated blockade of selectin interactions favors inhibition of $T$ cell activation/differentiation rather than lymphocyte rolling are our recent observations in the ischemic livers in which rPSGL-Ig therapy failed to affect the initial recruitment of $T$ lymphocytes into the liver grafts (AJ Coito, unpublished data). Indeed, a growing number of studies illustrate that other adhesion molecules can mediate leukocyte rolling. For example, the $\alpha 4 \beta 1$ integrin also supports leukocyte tethering and rolling in flow on VCAM-1 (Alon et al, 1995). Moreover, the $\alpha 4$ integrin can initiate leukocyte-endothelial cell interactions in the absence of selectins in vivo and, in IL-4-treated mice, the absence of selectins does not affect the recruitment of MNC cells in cremasteric postcapillary venules (Hickey et al, 1999). In our studies, VCAM-1 was up-regulated on the vascular cardiac endothelium independent of the rPSGL-Ig therapy. Furthermore, IL-4 was predominantly expressed in the rPSGL-Ig-treated grafts at 24 hours (data not shown). Interestingly, at 6 hours, MCP-1, a chemokine that specifically stimulates the directional migration of $T$ cells as well as monocytes, was found to be nearly absent in the cardiac grafts of rPSGL-Ig-treated rats. It has been postulated that MCP-1, a member of the $\mathrm{C}-\mathrm{C}$ chemokines, may play an important role in immune cell recruitment into sites of the antigenic challenge (Russell et al, 1993). The initial lack of MCP-1 observed in the rPSGL-lg-treated grafts might also represent one of the mechanisms by which this therapeutic approach effectively reduced the number of leukocytes at the graft site.

One of the most striking effects of rPSGL-Ig therapy was the apparent absence of myocardial infarcts in the treated hosts. Despite some MNC infiltration, the morphology of those grafts was largely preserved. There is a growing body of evidence that oxidative stress causes cardiac injury (Aikawa et al, 1997). Heme oxygenases, rate-limiting enzymes in the degradation of heme, catalyze the conversion of heme into biliverdin, carbon monoxide (CO), and free iron (Soares et al, 1998). CO increases cellular levels of cGMP, which regulates vascular tone and smooth muscle development and, as recently shown, has antiinflammatory effects (Otterbein et al, 2000). Bilirubin is known to be a potent antioxidant (Stocker et al, 1987). HO-1, also known as hsp32, is induced by a variety of cellular stresses, including oxygen deprivation and free radical-mediated stress. The overexpression of this enzyme exerts potent cytoprotective effects (Soares et al, 1998), consistent with our recent studies in steatotic rat liver models of I/R injury (Amersi et al, 1999). Moreover, it has been reported that $\mathrm{HO}-1$ has a critical role in survival of concordant mouse to rat cardiac transplants (Lin et al, 1999; Soares et al, 1998). We analyzed HO-1 induction in cardiac grafts on rPSGL-Ig therapy. This stress protein was almost absent at 6 hours and highly up-regulated by infiltrating MNC, primarily macrophages, at 24 hours after transplant. In contrast, $\mathrm{HO}-1$ expression was minimal in control grafts at both 6 hours and 24 hours. The virtual absence of $\mathrm{HO}-1$ expression in 24-hour control grafts might relate to the myocardial apoptosis observed in the graft infarcted areas. In an attempt to elucidate the role mediated by $\mathrm{HO}-1$ in our experimental settings we administered ZnPP, a known HO-1 antagonist, to recipients pretreated with rPSGL-lg. Interestingly, ZnPP administration resulted in significant increased MNC infiltration and activation. The numbers of intragraft T cells, particularly CD8+ cells, in the ZnPP-treated recipients were significantly higher than in recipients treated with rPSGL-Ig alone, whereas the levels of IL-2R + cells in the ZnPP-treated recipients were comparable with those observed in untreated controls. These findings support the concept that $\mathrm{HO}-1$ and/or its downstream mediators, such as $\mathrm{CO}$, are powerful antiinflammatory agents (Otterbein et al, 2000). Interestingly, we did not find significant differences in CD4+ cells and macrophages infiltrating the cardiac allografts treated with rPSGL-Ig alone or in combination with ZnPP. These observations suggest that HO-1 may account for some but not all of the effects of the rPSGL-1 therapy on leukocyte infiltration. Although it is important that in our experimental settings $\mathrm{ZnPP}$ is a potent inhibitor of $\mathrm{HO}-1$ activity, it never completely abolishes $\mathrm{HO}-1$ expression (Amersi et al, 1999; Katori et al, in press). Administration of ZnPP to rPSGL-lg-treated recipients has also led to a decrease in antiapoptotic Bag-1 protein expression. In addition, caspase-3 (p20) a proapoptotic protein that was absent in recipients conditioned with rPSGL-Ig, moderately increased at the graft site in recipients that received adjunctive $\mathrm{ZnPP}$. This evidence for the role of $\mathrm{HO}-1$ in the regulation of apoptotic protein networks is consistent with our recent data in the ischemic rat livers in which gene therapy-induced $\mathrm{HO}-1$ overexpression was as- 
sociated with up-regulation of antiapoptotic proteins (Coito, unpublished). In supporting our interpretation are recent findings that myocardial apoptosis occurs during myocardial infarction and that $\mathrm{HO}-1$ null mice are more prone to develop myocardial infarcts than their wild-type counterparts (Yet et al, 1999; Bialik et al, 1997).

In conclusion, the blockade of selectin-PSGL-1 interactions depresses intragraft expression of Th1 type cytokines and might inhibit the differentiation of Th1 type cells. In addition, it up-regulates HO-1 expression and protects against myocardial infarction and apoptosis. Hence, this study reports on the previously unrecognized role and mechanisms of selectin-PSGL-1-mediated interactions after in vivo alloantigenic challenge.

\section{Materials and Methods}

\section{Animals and Grafting Techniques}

Male inbred adult rats (Harlan Sprague-Dawley, Indianapolis, Indiana) were used. Lewis (LEW; RT') rats were recipients of cardiac allografts from Lewis $\times$ Brown Norway F1 hybrids (LBNF1; RT1 $1^{1 / n}$ ) and Brown Norway (BN; $\mathrm{RT}^{\mathrm{n}}$ ) rats served as a source of skin grafts (Kupiec-Weglinski et al, 1991). Orthotopic skin $(3 \times 3 \mathrm{~cm})$ grafts were placed bilaterally to defects on the dorsal thorax of prospective recipients (Day -7 ). Hearts were transplanted to the abdominal great vessels of the skin graft-sensitized recipients, and their function was monitored by palpation. Rejection was defined as the day of cessation of heartbeat and was confirmed by histology. All the surgical procedures were done under the guidelines of the UCLA Animal Research Committee.

\section{Treatment with rPSGL-Ig}

rPSGL-lg (Genetics Institute, Cambridge, Massachusetts) is a recombinant soluble form of PSGL-1 (Sako et al, 1995). This dimeric molecule consists of the amino terminal 47 a.a. and carbohydrate portion of mature PSGL-1 fused to the Fc portion of human IgG1 (Sako et al, 1995). rPSGL-lg, free of endotoxin, was administered intravenously to skin-presensitized cardiac allograft recipients ( $100 \mu \mathrm{g} / \mathrm{day} / \mathrm{rat})$ according to three protocols: (a) during the effector phase, that is, at Day 0 and Day +1 postcardiac engraftment $(n=5)$; (b) during the skin graft-mediated sensitization phase, that is, between Day -7 and Day $-1(n=11)$; and (c) during both sensitization and effector phases, that is, between Day -7 and Day $+2(n=6)$. The control group received PBS only $(n=10)$.

\section{Treatment with ZnPPn}

ZnPP was given at Day $0(1.5 \mathrm{mg} / \mathrm{kg}$ iv) to recipients previously treated with rPSGL-lg during the sensitization phase (Day -7 to Day -1 ). This therapy was based on our previous findings that ZnPP is a potent and fast inhibitor of HO-1 expression (Amersi et al, 1999; Katori et al, in press). For instance, in a model of heart $\mathrm{I} / \mathrm{R}$, a single dose of $\mathrm{ZnPP}$ given at reperfusion to CoPP-treated grafts reduces HO-1 enzymatic activity to control levels as earlier as 4 minutes after transplantation (Katori et al, in press).

\section{Serum IgM/IgG Allo-Ab Responses}

The circulating donor-specific allo-Ab responses were screened in the recipient's serum as described (Wasowska et al, 1996). Briefly, cervical BN lymph node target cells were first incubated with serially diluted (1/4 to 1/128) heat-inactivated experimental serum samples for 45 minutes at $4^{\circ} \mathrm{C}$. To stain for $\operatorname{lgG}$ and $\operatorname{lgM}$, the cells were reacted with a mixture of FITCconjugated goat anti-rat IgG (Fc specific) and PEconjugated goat anti-rat IgM ( $\mu$-chain) (Jackson Immunoresearch, West Grove, Pennsylvania) for 30 minutes at $4^{\circ} \mathrm{C}$. The cells were then washed, fixed in $1 \%$ neutral buffered formalin, and analyzed on an EPICS C cell sorter (Coulter, Hialeah, Florida).

\section{Intragraft Cell Infiltration}

Hematoxylin/eosin and immunoperoxidase staining were used for serial assessment of myocardial/vascular preservation and detection of inflammatory cell infiltration/activation, adhesion molecules, and HO-1 expression (Coito et al, 1995). The presence of cytokine gene products was demonstrated by the saponin-immunoperoxidase method as previously described (Coito et al, 2000). Antibodies against human $\mathrm{P}$-selectin (CD62P, cross reacts with rat P-selectin; Pharmingen, San Diego, California), and rat VCAM-1 (CD106; courtesy of Dr. R. Lobb, Biogen, Cambridge, Massachusetts), ICAM-1 (CD54; Harlan Bioproducts for Science, Indianapolis, Indiana), T cells (TCR- $\alpha / \beta$ chains, R73; Harlan), monocytes/macrophages (ED1; Harlan), CD25 (IL-2R; ART-18; courtesy of Dr. T. Diamantstein, Berlin, Germany), HO-1 (does not react with HO-2; StressGen Biotechnologies, Victoria, B.C., Canada), IL-2 (R\&D Systems, Minneapolis, Minnesota), IFN- $\gamma$ (DB-1; Harlan), RANTES and MCP1 (Pepro Tech, Rocky Hill, New Jersey) were used. The sections were evaluated blindly by counting the labeled cells in triplicates within 10 high-power fields (HPF)/section. Some antigens were analyzed in a semiquantitative fashion, and the relative abundance of each was judged as $(-)$ negative, $( \pm)$ minimal, $(+)$ little, $(++)$ moderately abundant, or $(+++)$ very abundant.

\section{Intragraft Cytokine Gene Expression}

RNA from cardiac grafts was prepared by the guanidinium thiocyanate-cesium chloride method and competitive template (CT) RT-PCR was performed, as described (Siegling et al, 1994). Quantification of cytokine cDNA derived from this RNA was carried out using multispecific control fragment as an internal standard for competitive PCR. The sense and antisense primer sequences derived from rat CD3, CD25, IL-2, IFN- $\gamma$, IL-10, RANTES, MCP-1, and $\beta$-actin CDNA were used (Nadeau et al, 1995; Siegling et al, 1994). 


\section{Intragraft HO-1 and Bag-1 Protein Expression}

Proteins extracted from cardiac samples (60 $\mu \mathrm{g} / \mathrm{sam}$ ple) were subjected to $12 \%$ SDS-PAGE and transferred to nitrocellulose membranes (Bio-Rad Laboratories, Hercules, California), as described (Ke et al, 2000). Gels were then stained with Coomassie blue to document equal protein loading. Membranes were blocked ( $>2$ hours) in 5\% defatted dry milk/PBS/0.1\% Tween-20 and incubated with anti-HO-1 (Sangstat, San Francisco, California), anti-Bag-1 (C-16), anticaspase-3 (H-277), or the control anti-actin (I-19) (Santa Cruz Biotechnology, Santa Cruz, California) antibodies. After 1 hour of incubation with the primary $\mathrm{Ab}$, blots were washed three times (PBS/0.1\% Tween), and the secondary, peroxidase-conjugated Ab (Pierce, Rockford, Illinois) was added for another hour. Finally, the blots were washed three times (PBS/ $0.1 \%$ Tween) and immunoreactive proteins were visualized using Western blot chemiluminescent detection (Amersham Pharmacia Biotech, Piscataway, New Jersey). Relative quantities of proteins were determined using a densitometer (Kodak Digital Science 1D Analysis Software, Rochester, New York).

\section{Intragraft Apoptosis}

Apoptosis was detected by TUNEL. This tailing reaction is especially sensitive to the type of DNA fragmentation occurring in apoptotic rather than necrotic cell death (Gold et al, 1994). The TUNEL assay was carried out on $5-\mu \mathrm{m}$ cryostat sections using the TACS 2 TdT-DAB in situ Apoptosis Detection Kit (Trevigen, Gaithersburg, Maryland), following the manufacture's instructions. TUNEL+ cells were detected under light microscopy.

\section{References}

Aikawa R, Komuro I, Yamazaki T, Zou Y, Kudoh S, Tanaka M, Shiojima I, Hiroi Y, and Yazaki Y (1997). Oxidative stress activates extracellular signal-regulated kinases through Src and Ras in cultured cardiac myocytes of neonatal rats. J Clin Invest 100:1813-1821.

Alon R, Kassner PD, Carr MW, Finger EB, Hemler ME, and Springer TA (1995). The integrin VLA-4 supports tethering and rolling in flow on VCAM-1. J Cell Biol 128:1243-1253.

Amersi F, Buelow R, Kato H, Ke B, Coito AJ, Shen XD, Zhao D, Zaky J, Melinek J, Lassman CR, Kolls JK, Alam J, Ritter T, Volk HD, Farmer DG, Ghobrial RM, Busuttil RW, and KupiecWeglinski JW (1999). Upregulation of heme oxygenase-1 protects genetically fat zucker rat livers from ischemia/ reperfusion injury. J Clin Invest 104:1631-1639.

Austrup F, Vestweber D, Borges E, Lohning M, Brauer R, Herz U, Renz H, Hallmann R, Scheffold A, Radbruch A, and Hamann A (1997). P- and E-selectin mediate recruitment of T-helper-1 but not T-helper-2 cells into inflamed tissues. Nature 385:81-83.

Bialik S, Geenen DL, Sasson IE, Cheng R, Horner JW, Evans SM, Lord EM, Koch CJ, and Kitsis RN (1997). Myocyte apoptosis during acute myocardial infarction in the mouse localizes to hypoxic regions but occurs independently of p53. $\mathrm{J}$ Clin Invest 100:1363-1372.
Coito AJ, Binder J, Brown LF, de Sousa M, Van de Water L, and Kupiec-Weglinski JW (1995). Anti-TNF-alpha treatment down-regulates the expression of fibronectin and decreases cellular infiltration of cardiac allografts in rats. J Immunol 154:2949-2958.

Coito AJ, de Sousa M, and Kupiec-Weglinski JW (1994). The role of cellular and extracellular matrix adhesion proteins in organ transplantation. Cell Adhes Commun 2:249-255

Coito AJ, Onodera K, Kato H, Busuttil RW, and KupiecWeglinski JW (2000). Fibronectin-mononuclear cell interactions regulate type 1 helper T cell cytokine network in tolerant transplant recipients. Am J Pathol 157:1207-1218.

Dulkanchainun TS, Goss JA, Imagawa DK, Shaw GD, Anselmo DM, Kaldas F, Wang T, Zhao D, Busuttil AA, Kato H, Murray NG, Kupiec-Weglinski JW, and Busuttil RW (1998). Reduction of hepatic ischemia/reperfusion injury by a soluble P-selectin glycoprotein ligand-1. Ann Surg 227:832-840.

Frenette PS, Mayadas TN, Rayburn H, Hynes RO, and Wagner DD (1996). Double knockout highlights value of endothelial selectins. Immunol Today 17:205.

Fuhlbrigge RC, Kieffer JD, Armerding D, and Kupper TS (1997). Cutaneous lymphocyte antigen is a specialized form of PSGL-1 expressed on skin-homing T cells. Nature 389: 978-981.

Gold R, Schmied M, Giegerich G, Breitschopf H, Hartung HP, Toyka KV, and Lassmann H (1994). Differentiation between cellular apoptosis and necrosis by the combined use of in situ tailing and nick translation techniques [see comments]. Lab Invest 71:219-225.

Hickey MJ, Granger DN, and Kubes P (1999). Molecular mechanisms underlying IL-4-induced leukocyte recruitment in vivo: A critical role for the alpha 4 integrin. J Immunol 163:3441-3448.

Homeister JW, Zhang M, Frenette PS, Hynes RO, Wagner DD, Lowe JB, and Marks RM (1998). Overlapping functions of $\mathrm{E}-$ and P-selectin in neutrophil recruitment during acute inflammation. Blood 92:2345-2352.

Katori M, Buelow R, Ke B, Ma J, Coito AJ, lyer S, Southard D, Busuttil RW, and Kupiec-Weglinski JW (In press, 2001). Heme oxygenase-1 overexpression protects rat hearts from cold ischemia/reperfusion injury via anti-apoptotic pathway. Transplantation.

Ke B, Coito AJ, Kato H, Zhai Y, Wang T, Sawitzki B, Seu P, Busuttil RW, and Kupiec-Weglinski JW (2000). Fas ligand gene transfer prolongs rat renal allograft survival and downregulates anti-apoptotic Bag-1 in parallel with enhanced Th2-type cytokine expression. Transplantation 69:16901694.

Kupiec-Weglinski JW, Sablinski T, Hancock WW, Di Stefano R, Mariani G, Mix CT, and Tilney NL (1991). Modulation of accelerated rejection of cardiac allografts in sensitized rats by anti-interleukin 2 receptor monoclonal antibody and cyclosporine therapy. Transplantation 51:300-305.

Lim YC, Henault L, Wagers AJ, Kansas GS, Luscinskas FW, and Lichtman $\mathrm{AH}$ (1999). Expression of functional selectin ligands on Th cells is differentially regulated by IL-12 and IL-4. J Immunol 162:3193-3201.

Lin Y, Soares MP, Sato K, Takigami K, Csizmadia E, Smith N, and Bach FH (1999). Accommodated xenografts survive in the presence of anti-donor antibodies and complement that 
precipitate rejection of naive xenografts. J Immunol 163: 2850-2857.

McEver RP and Cummings RD (1997). Role of PSGL-1 binding to selectins in leukocyte recruitment. J Clin Invest 100:S97-103.

Nadeau KC, Azuma H, and Tilney NL (1995). Sequential cytokine dynamics in chronic rejection of rat renal allografts: Roles for cytokines RANTES and MCP-1. Proc Natl Acad Sci USA 92:8729-8733.

Otterbein LE, Bach FH, Alam J, Soares M, Tao LH, Wysk M, Davis RJ, Flavell RA, and Choi AM (2000). Carbon monoxide has anti-inflammatory effects involving the mitogen-activated protein kinase pathway. Nat Med 6:422-428.

Russell ME, Adams DH, Wyner LR, Yamashita Y, Halnon NJ, and Karnovsky MJ (1993). Early and persistent induction of monocyte chemoattractant protein 1 in rat cardiac allografts. Proc Natl Acad Sci USA 90:6086-6090.

Sako D, Comess KM, Barone KM, Camphausen RT, Cumming DA, and Shaw GD (1995). A sulfated peptide segment at the amino terminus of PSGL-1 is critical for P-selectin binding. Cell 83:323-331.

Siegling A, Lehmann M, Platzer C, Emmrich F, and Volk HD (1994). A novel multispecific competitor fragment for quantitative PCR analysis of cytokine gene expression in rats. J Immunol Methods 177:23-28.

Soares MP, Lin Y, Anrather J, Csizmadia E, Takigami K, Sato K, Grey ST, Colvin RB, Choi AM, Poss KD, and Bach FH (1998). Expression of heme oxygenase-1 can determine cardiac xenograft survival. Nat Med 4:1073-1077.
Stocker R, Yamamoto Y, McDonagh AF, Glazer AN, and Ames BN (1987). Bilirubin is an antioxidant of possible physiological importance. Science 235:1043-1046.

Takada M, Nadeau KC, Shaw GD, Marquette KA, and Tilney NL (1997). The cytokine-adhesion molecule cascade in ischemia/reperfusion injury of the rat kidney: Inhibition by a soluble P-selectin ligand. J Clin Invest 99:2682-2690.

Wagers AJ, Waters CM, Stoolman LM, and Kansas GS (1998). Interleukin 12 and interleukin 4 control T cell adhesion to endothelial selectins through opposite effects on alpha1, 3-fucosyltransferase VII gene expression. J Exp Med 188: 2225-2231.

Wasowska B, Wieder KJ, Hancock WW, Zheng XX, Berse B, Binder J, Strom TB, and Kupiec-Weglinski JW (1996). Cytokine and alloantibody networks in long term cardiac allografts in rat recipients treated with rapamycin. J Immunol 156:395404.

Yet SF, Perrella MA, Layne MD, Hsieh CM, Maemura K, Kobzik L, Wiesel P, Christou H, Kourembanas S, and Lee ME (1999). Hypoxia induces severe right ventricular dilatation and infarction in heme oxygenase- 1 null mice. J Clin Invest 103:R23-R29.

Zhai Y and Kupiec-Weglinski JW (1999). What is the role of regulatory $\mathrm{T}$ cells in transplantation tolerance? Curr Opin Immunol 11:497-503. 